INTERNATIONAL JOURNAL OF RESEARCHES IN BIOSCIENCES, AGRICULTURE AND TECHNOLOGY

(C) VISHWASHANTI MULTIPURPOSE SOCIETY (Global Peace Multipurpose Society) R. No.MH-659/13(N) www.vmsindia.org

\title{
SURVEY ON WIRELESS BODY AREA NETWORK FOR HEALTHCARE APPLICATIONS
}

\section{S. M. Ghatole ${ }^{1}$ and P. B. Dahikar ${ }^{2}$}

${ }_{1}^{1}$ Department of Electronics, Shivaji Science College, Nagpur (MS) India. ${ }^{2}$ Department of Electronics, Kamala Nehru Science College, Nagpur (MS) India. smghatole@gmail.com

\begin{abstract}
:
This paper presents the survey/review on various aspects of WBAN technologies that are being used in healthcare applications. Wireless Body Area Network (WBAN) is the network of sensors worn on the body, used to describe a network of devices connected to wireless communication system. In particular, we have inspected monitoring and sensing, system architectures and applications of Wireless Body Area Network for healthcare system. We conclude by discussions on open research issues, their potential solutions and future trends useful for applications in healthcare system. WBAN opens up a whole new field of sensor networking and intelligent technology for healthcare applications but the problem lies in varying specifications and operating platforms.
\end{abstract}

Keywords: Wireless body, area network, body sensors, healthcare, WBAN survey.

\section{Introduction:}

Twenty first century is the age of Information and Communication Technology, the use of this technology for processing information has become inherent part of human life. In this new era of enormously growing population, lack of medication facility and the rapidly increasing health problems with modern lifestyle require health monitoring for endurance of especially elders, baby and chronically ill patients. An aging population and sedentary lifestyle are fueling the prevalence of chronic diseases such as cardiovascular diseases, hypertension, and diabetes. According to the WHO, cardiovascular disease causes 30 percent of all deaths in the world. Diabetes currently affects 180 million people worldwide and is expected to affect around 360 million by 2030. More than 2.3 billion people will be overweight by 2015. A rapid rise in debilitating neuro-degenerative diseases such as Parkinson's and Alzheimer's is threatening millions more [1].

Recently, Wireless Body Area Network (WBAN) is growing research area in the healthcare industry across the globe. The difficulty mainly concerns with the delivery of effective and efficient services to a population where chronic and acute diseases are most prevalent. Healthcare services are gradually shifted from delicate hospital care to outpatient care to home care. Home care, typically involves periodic visits by a nurse or other caregivers, and may require the maintenance of detailed records about their diet, and health. This situation results in a drastic change in the availability, accessibility and affordability of healthcare. It needs to be noted that the healthcare providers are in an advantageous position where they can use the best of technology to deliver the requirement. It would be absolutely impracticable though to think that technology alone can fulfill such a persistent need. The patient monitoring market is an expanding and profitable segment in the global healthcare industry which needs to be addressed and captured. The Rise in healthcare costs, short of healthcare availability and the increase in average life expectancy of individuals in many developed nations represent the primary motivating factors for innovation in healthcare. One such innovation is the continuous monitoring of patients via sensors connected as a WBAN. This will enable improved analysis and modification of treatment regimes, computer assisted rehabilitation and early detection of life threatening trial. The probability of user acceptance of WBAN technologies has increased with advances in trimness of electronic devices, sensing, battery and wireless communication technologies. The key elements of a WBAN are smart miniaturized devices that are able to sense, process and communicate the signal information. The system is designed such that they can be worn or implanted, and is able to monitor and transmit physiological signals to dedicated medical servers without much interference to the daily routine of the patient. We review monitoring and sensing devices used in WBANs [2]. It requires the representation about an inspection of power efficient protocols. The WBAN system architecture is review completely. An approach to routes in WBAN, various security techniques and protocols are inspected. Finally, we conclude with a discussion of open research problems and future trends in healthcare system.

\section{Wireless Body Area Network (WBAN):}

A wireless body area network (WBAN) connects independent nodes (e.g. sensors and actuators) that are situated in the clothes, on 
the body or under the skin of a person. The network typically extends over the whole human body and the nodes are connected through a wireless communication channel. According to the implementation, these nodes are placed in a star or multi hop topology. A WBAN offers many promising, new applications in home/health care, medicine, sports, multimedia, and many other areas, all of which make advantage of the unconstrained freedom of movement a WBAN offers. A Wireless Body Area Network (WBAN) consists of several small devices close to, attached to or implanted into the human body. These devices communicate by means of a wireless network. Interaction with the user or other persons is generally handled by a central device, e.g. a PDA. The lowest level consist a set of intelligent sensors or nodes. These are the reduced function devices. These can only communicate with the parent device and cannot act as parent. The second level is the personal server (Internet enabled PDA, cell-phone, or home computer). These are full function devices and they can communicate with their children as well as with the external network. The third level encompasses a network of remote server which is the remote application to which data or information is transferred [3].

\section{Sensor categories:}

Sensors fall into three categories:

1. Physiological sensors:

It is used for measuring ambulatory blood pressure, continuous glucose monitoring, core body temperature, blood oxygen, and signals related to respiratory inductive plethysmography, electrocardiography (ECG), electroencephalography (EEG), and electromyography (EMG).

\section{Biokinetic sensors:}

It measures acceleration and angular rate of rotation derived from human movement.

\section{Ambient sensors:}

It is used to measure environmental phenomena, such as humidity, light, sound pressure level, and temperature.

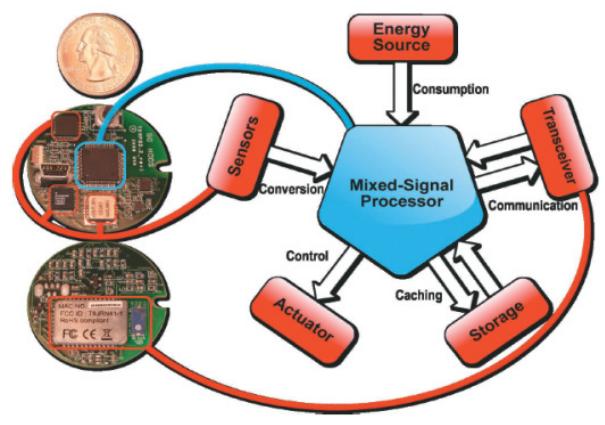

\section{BASN node architecture:}

The advent of miniaturized sensors and actuators for monitoring, diagnostic, and therapeutic functions, and advances in wireless technology have opened up new frontiers in the race to conquer healthcare challenges. Ultralow-power wireless connectivity among devices placed in, on, and around the human body is seen as a key technology enabling unprecedented portability for monitoring physiological signs in the hospital, at home, and on the move [4]. Although the architectural components are similar to those of a typical wireless sensor network node, a BASN node presents unique challenges and opportunities from sensing to communication. A BASN can interact with existing systems, such as networks in hospitals and retirement communities. Body sensors in BASN nodes provide data to the body aggregator, which is central to managing body events. Body aggregators perform a multitude of functions, including sensing, fusing data from sensors across the body, serving as a user interface, and bridging BASNs to higher-level infrastructures and thus to other stakeholders. Wearable and implantable sensors are used for sensing and monitoring in WBANs. The biological parameters are the measure of knowing the health condition of patients. The wearable sensors are used to monitor several physiological parameters. The data gathered from these sensors is sent to a data accumulator. The data accumulator then forwards it to the medical server. The personal server sends out message to the doctor's mobile phone in occurrence of life-threaten circumstances through GSM network. The sensor network provides biological information and on the basis of assessment, assistance and treatment is decided. [5].

\section{WBAN Application Areas:}

- Body senor network

- Fitness monitoring

- Wearable audio

- Mobile device centric

- Gaming \& Entertainment

- Consumer Electronics Applications : media players, and headsets

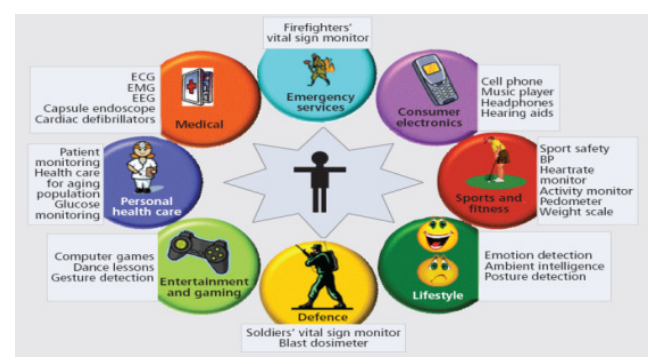


Body Sensor Network plays versatile role in Medical applications.

$\begin{array}{ll}\text { - } & \text { Pearable Monitoring Systems } \\ \text { - } & \text { Electrocardiograph (EKG) } \\ \text { - Electroencephalograph (EEG) } \\ \text { - Electromyograph (EMG) }\end{array}$

\section{Sensing and Monitoring:}

This section represents the WBAN sensors used in healthcare system. Wearable and implantable sensors are generally used for sensing and monitoring in WBANs. The biological parameters are the measure of knowing the health condition of patients. The wearable sensors are used to monitor several physiological parameters. A biomedical device, pulse oximeter is used indirectly for the measurements of the oxygen saturation levels (SpO2) in an individual's blood. It also detects the changes in blood volume in the skin that coincide with the cardiac cycle. The quasiperiodic signal that comes out as output is called a photoplethysmograph (PPG), and used to determine heart-rate. A wearable PPG biosensor in the form of a ring has been developed by Yang and Rhee [6].

A wearable ECG sensor for WBAN has been described in [7]. The electrocardiogram (ECG) is a waveform that represents the propagation of electric potentials through the heart muscle with respect to time. Therefore, the ECG waveform provides a non-invasive means for investigating heart function. Fulford-Jones et.al. designed an ECG sensor that is supported by a Mica 2 mote hardware platform]. A network of wearable wireless blood pressure monitors has been studied in [8]. It has been observed that ambulatory blood pressure (BP) is more closely related to target organ damage and cardiovascular events than BP readings taken in a clinical environment. This fact provides the motivation for the creation of wireless BP sensors. Poon et. al. have created a cuff-less BP watch sensor, based on the pulse transit time (PTT) method for measuring BP [9].

Wearable sensors have also been used for activity/motion detection. The level of activity or the nature of motion of an individual can be detected by a system that combines an accelerometer with a gyroscope. An example of an integrated accelerometer/gyroscope is presented in [10]. An EEG monitor as a wearable sensor has been described in [11]. Electroencephalography (EEG) is a representation of the electrical activity of the brain. Farshchiet. al. have introduced a wireless neural interface, using Mica2 and Mica2dot systems as the wireless sensor platforms, which is capable of acquiring two channels of EEG data.

Implantable sensors have been studied in [12], implantable neural stimulators send electrical impulses into the brain or spinal cord for the treatment of Parkinson disease, intractable epilepsy and chronic pain. On body medical sensors are wearable or installable physiological sensors like EEG, ECG, Heartbeat, Blood pressure, Oximeter, etc. In-body or implanted medical sensors are used for convenience to provide the mobility and normal lifestyle to patients. The implanted sensors are more precise and responsive to varying condition of a patient. Some of the implanted sensors are cardiac treatment through pacemakers, defibrillators, insulin pumps, glucose and blood pressure monitoring, etc. Present WBAN technology is mostly developed on-demand; WBANs are developed in response to specific physiological requirements. Using dynamic programming environments and cognitive interfaces, we should be able to measure multiple parameters in the human body and use this data to aid in preventive medicine and diagnosis. Since WBANs comprise of networks of sensors performing specialized detection of physiological data, the cost and size of these devices impose limitations on their use. Creating networks that seamlessly interface with the human environment will be an interesting area of research [13].

\section{Conclusion:}

WBAN opens up a whole new field of sensor networking and intelligent technology. It is a very practical way to track user activities for different purposes. It has a wide range of implementations in Medical rehabilitation, digital IDs, military and ultimately to personal entertainment systems. There are some design and social issues which are currently posing some limitation on commercial level implementation of WBAN.

The sensors used by the medical community are manufactured by a number of developers hence they vary in specifications and operating platforms. A major issue is to provide interoperable environments in heterogeneous sensor networks. Hence service oriented middleware for wireless sensor networks is required to provide common interfaces to utilize sensor nodes irrespective of sensor node platform. There is need for standardization and the sensor middleware is implemented as a Web 
service which defines standard interfaces to interact with client applications. A service oriented middleware for wireless sensor networks is needed to provide interoperability.

\section{References:}

[1] World Health Organization [online] http://www.who.int/mediacentre/factsheets / fs 317/en/index.html.

[2] S. M. Ghatole, K. Y. Rokde, S. S. Shende, P. B. Dahikar, "Healthcare System with interactive Biosensors" International Journal of Electronics, Communication \& Soft Computing Science and Engineering, Volume 1, Issue 1, June 2015.

[3] K. Y. Rokde, S. M. Ghatole, S. S. Shende, P. B. Dahikar, "An Embedded System for Patient Heartbeat Monitoring" International Journal of Electronics, Communication \& Soft Computing Science and Engineering, Volume 1, Issue 1, June 2015 .

[4] Mark A. Hanson, Harry C. Powell Jr., Adam T. Barth, Kyle Ring genberg, Benton H. Calhoun, James H. Aylor, and John Lach, Body Area Sensor Networks: Challenges and Opportunities, University of Virginia, IEEE Computer Society2009

[5] S. M. Ghatole, K. Y. Rokde, S. S. Shende, P. B. Dahikar, "Role of Wireless Body Area Network inRemote Healthcare Monitoring" International Journal of Researches in Biosciences, Agriculture and Technology (IJRBAT), Vol. II, Issue (7), Nov 2015: ISSN 2347 - 517X, 154-157.

[6]B. H. Yang and S. Rhee, "Development of the ring sensor for healthcare automation," Robotics and Autonomous Systems, vol. 30, pp. 273-281, 2000.
[7]T. R F. Fulford-Jones, et al., "A Portable, LowPower, Wireless Two-Lead EKG System," in 26th Annual International Conference of the IEEE EMBS San Francisco, CA, USA, 2004, pp. 2141-2144.

[8] K. Kario, et al. (2003) Ambulatory Blood Pressure Monitoring for Cardiovascular Medicine. IEEE Engineering in Medicine and Biology Magazine.81-88.

[9] C. C. Y. Porn, et al., "M-Health: The Development of Cuff-less and Wearable Blood Pressure Meters for Use in Body Sensor Networks," in Proceedings of IEEE/NLM Le Science Systems and Applications Workshop, 2006.

[10] C. Shnayder, et al., "Sensor Networks for Medical Care," Technical Report TR-08-05, Division of Engineering and Applied Sciences, Harvard University, 2005.

[11] F. Farshchi, et al., "A Tiny OS-Based Wireless Neural Interface," in Proceedings of the 26th Annual International Conference of the IEEE EMBS, 2004.

[12] M. Ghovanloo, et al., "A BiCMOS Wireless Interface Chip for Micro machined Stimulating Microprobes," in Proceedings of IEEE-EMBS Special Topic Conference on crotechnologies in Medicine and Biology, 2002, pp. 277-282.

[13] Ming Li And Wenjing Lou, Data Security And Privacy In Wireless Body Area Networks, Worcester Polytechnic Institute KuiRen, Illinois Institute Of Technology, IEEE Wireless Communications • February 2010.

[14] S. M. Ghatole, K. Y. Rokde, P. B. Dahikar, Zig Bee:A Wireless Communication Network Kamla Nehru Journal of Science \& Technology (KNJST) Vol.- 1 ISBN:978-93-81432-97-6, 62-66. 